\title{
PHYSICAL INTERPRETATION OF THE SEPARATION CRITERIA
}

\author{
I.J. LIN AND A. KATYSHEV \\ Minerals Engineering Research Centre, Technion, Haifa 32000, \\ Israel
}

(Received July 5, 1995, in the final form August 19, 1995)

\begin{abstract}
This work considers a separation process and the attendant losses on the basis of thermodynamic analogies. Energy-oriented efficiency as a criterion of the process, and the entropy-oriented efficiency as a criterion of its results, are compared. An example shows that the values are close to each other. As a practical recommendation, a modification of the feeder system is suggested.
\end{abstract}

\section{INTRODUCTION}

The results of separation can be presented in a form of cumulative curves: recovery vs. grade, or mass fraction vs. grade. As was shown in [1], neither recovery nor grade can serve as separation criteria by themselves, but only in combination which opens a way to a wide variety of possible criteria.

Barari, Sen and Gupta [2] reviewed measures of the separation efficiency, one of the best known of which is the Hancock criterion

$$
\eta=\frac{\epsilon_{\mathrm{c}}-\gamma_{\mathrm{c}}}{1-\alpha}
$$

where $\alpha, \epsilon_{\mathrm{c}}$ and $\gamma_{\mathrm{c}}$ are the initial grade, recovery and mass fraction of the concentrate, respectively. 
Rony [4] suggested a criterion covering all components and all products:

$$
\eta=\left[\begin{array}{ll}
\epsilon_{11} \ldots \ldots \ldots & \epsilon_{1 \mathrm{n}} \\
\vdots & \vdots \\
\vdots & \vdots \\
\epsilon_{\mathrm{p} 1} \ldots \ldots \ldots & \epsilon_{\mathrm{pn}}
\end{array}\right]
$$

Other suggestions are those of Petho and Tompos [4] (Tromp distribution curves) and De Klerk and Cloete [5] (entropy difference.)

The present work deals with the physical meaning of criteria constructed on the basis of energy and entropy changes during the separation process.

\section{THEORETICAL CONSIDERATIONS}

\section{Work of the Separation Force}

A two-component mixture is in equilibrium: minimum potential energy and maximum entropy from which the system deviates during separation. Physically, separation can be described as a deviation of particles from some middle point according to their separation properties (charge, mass, density etc.), under the action of force $F[6]$ :

$$
\mathbf{x}=\frac{\mathbf{F} \tau^{2}}{2 \mathrm{~m}}
$$

where $x$ is the deviation, $F$ is the separation force, $\tau$ is the separation time and $m$ is the mass of the particle. $F$ depends on property $q$, and on the intensity of the separation field $E$ (electrostatic, gravity, magnetic etc.):

$$
\mathrm{F}=\mathrm{qE}
$$

The summed property $Q$ ( $Q=q N, N$ being the number of particles) can be expressed as:

$$
\mathrm{Q}=\int \sigma(\mathrm{m}) \rho_{\sigma}(\mathrm{m}) \mathrm{dm}
$$


where $\sigma$ is the value of $Q$ per unit mass, $\rho_{\sigma}$ is the distribution function of $\sigma$.

The ideal distribution of a property is then given by

$$
\rho_{\sigma}=\alpha \delta\left(\sigma_{1}\right)+(1-\alpha) \delta\left(\sigma_{2}\right)
$$

The completely unseparable case is:

$$
\rho_{\sigma}=\delta\left(\sigma_{0}\right)
$$

where $\delta$ is the Dirac delta function.

Complete separation is impossible because of the non-deal distribution of the properties and the losses involved in the separation process.

The work of the separation force is;

$$
A=\Sigma F_{i} x_{i}
$$

Substituting eqs. (3) and (4) in eq. (8) we obtain:

$$
\mathrm{A}=\frac{\mathrm{E}^{2} \tau^{2}}{2} \Sigma \mathrm{q}_{\mathrm{i}} \sigma_{\mathrm{i}}
$$

In a continuous form, by applying eq. (5) we get:

$$
\mathrm{A}=\frac{\mathrm{E}^{2} \tau^{2}}{2} \int\left(\sigma-\sigma_{0}\right)^{2} \rho(\mathrm{m}) \mathrm{dm}
$$

where the expression under the integral sign is the spread of the distribution. Denoting the integral as $\mathrm{D}_{\sigma}^{2}$ we have:

$$
\mathrm{A}=\frac{\left(\mathrm{E} \tau \mathrm{D}_{\sigma}\right)^{2}}{2}
$$


Since $\left(\mathrm{E} \tau \mathrm{D}_{\sigma}\right)^{2}$ represents the maximum velocity squared, in the direction of separation, averaged over all particles, eq. (11) is the loss of energy conservation:

$$
\mathrm{A}=\Sigma \frac{\mathrm{m}_{\mathrm{i}} \mathrm{v}_{1 \max }^{2}}{2}
$$

or, in a continuous form:

$$
\mathrm{A}=\frac{\mathrm{M}}{2} \int \mathrm{v}_{\max }^{2} \rho_{\mathrm{v}}(\mathrm{m}) \mathrm{dm}
$$

where $M$ is the total mass of the material.

Assuming that the deflection of the property per unit mass $\left(\sigma-\sigma_{0}\right)$ from the average depends linearly only on its grade:

$$
\sigma-\sigma_{0}=\sigma^{\star}\left(\beta_{\mathrm{p}}-\alpha\right)
$$

From eqs. (10) and (12) we thus get:

$$
\mathrm{A}=\frac{\mathrm{E}^{2} \tau^{2} \sigma^{2 \star}}{2} \int\left(\beta_{\mathrm{p}}-\alpha\right) \gamma(\mathrm{m}) \mathrm{dm}
$$

where $\gamma(\mathrm{m})$ is the mass fraction of particles with grade $\beta_{\mathrm{p}}$. This can be rewritten in the Stiltjes integral form:

$$
\mathrm{A}=\frac{\mathrm{E}^{2} \tau^{2} \sigma^{\star 2}}{2} \mathrm{M} \int\left(\beta_{\mathrm{p}}-\alpha\right)^{2} \mathrm{~d} \Gamma
$$

where $\Gamma(\mathrm{m})$ is the cumulative mass fraction.

Denoting $\mathrm{E} \tau \sigma^{\star}=\mathrm{v}_{\max }^{\star}$, we have:

$$
\mathrm{A}=\frac{\mathrm{Mv}_{\max }^{\star 2}}{2} \int\left(\beta_{\mathrm{p}}-\alpha\right)^{2} \mathrm{~d} \Gamma
$$


Let $\epsilon$ be the fraction of the useful mineral with the grade lower than $\beta_{\mathrm{p}}$. Then:

$$
\beta_{\mathrm{p}}=\frac{\mathrm{d} \epsilon_{\mathrm{p}}}{\alpha \mathrm{d} \Gamma}
$$

The work $A$ can be expressed as:

$$
\mathrm{A}=\frac{\mathrm{Mv}_{\mathrm{max}}^{\star_{2}}}{2} \alpha \int(\beta-\alpha) \mathrm{d} \epsilon_{\mathrm{p}}
$$

We can thus see that the work of the separation force is a function of liberation of the material.

\section{The Random Process}

Until now we have considered determinate processes, i.e. processes with a position of each particle after separation rigorously dictated by its grade. In practice, however, the assumption (14) is not invariably valid and there is a random effect as a result if which the destination of a particle is distributed about a middle point, even in the absence of an external field.

The random process can be characterised by the distribution of the particle velocity in the direction of the separation, similar to the Maxwell distribution for molecules. Assuming that a component of the random velocity is time-invariant, it can be described by a distribution of the inlet velocity. The random kinetic energy can be expressed as:

$$
\mathrm{W}_{\mathrm{r}}=\frac{1}{2} \mathrm{mv}_{\mathrm{r}}^{2}
$$

where $v_{\mathrm{r}}$ is the average velocity of a particle.

In analogy with the thermodynamic theory, motion of a particle can be considered as a random motion of molecules. The energy of the random process is thus:

$$
\mathrm{W}_{\mathrm{r}}=\frac{3}{2} \mathrm{kT}
$$


where $k$ and $T$ are parameters with the dimensions of the Boltzmann constant and temperature, respectively. Under the assumption of time-invariance of the random process, the process can be considered "isothermal",

$$
\mathrm{T}=\text { const }
$$

The random process is not grade-selective and thus tends to mix particles. Accordingly, it is why it can be considered as a source of losses, causing the entropy to increase.

The random velocity depends on a design of the feed, on the properties of the medium (viscosity etc.), and on the shape and mass of the particles. The larger is the mass of the particles, the weaker is the effect of the random process [1], which disposes each point of the final distribution of the separation about the corresponding point of the liberation function.

The deviation $x_{\mathrm{r}}$ under the process is given by:

$$
\mathrm{x}_{\mathrm{r}}=\mathrm{v}_{\mathrm{r}} \tau
$$

and its distribution being:

$$
\rho_{\mathrm{xr}}=\left(\mathrm{x}_{\mathrm{r}}\right)=\frac{\mathrm{dP}}{\mathrm{dx}_{\mathrm{r}}}=\frac{\mathrm{dP}}{\tau \mathrm{d} \mathrm{v}_{\mathrm{r}}}=\frac{1}{\tau} \rho_{\mathrm{vr}}
$$

where $P$ is the dimensionless cumulative distribution.

The corresponding value under the influence of the separation force is given by:

$$
\rho\left(\mathrm{x}_{\mathrm{r}}\right)=\frac{1}{\mathrm{v}^{\star} \tau} \rho_{\beta_{\mathrm{p}}}\left(\beta_{\mathrm{p}}\right)
$$

and the combined result under the influence of both processes is given by the convolution:

$$
\rho_{\mathrm{x}}(\mathrm{x})=\int \rho_{\sigma}\left(\mathrm{x}_{\sigma}\right) \rho_{\mathrm{r}}\left(\mathrm{x}-\mathrm{x}_{\sigma}\right) \mathrm{dx} \sigma
$$


whence by eqs. (24) and (25) we have:

$$
\rho_{\mathbf{x}}(\mathbf{x})=\frac{1}{\tau} \int \rho_{\mathrm{vr}}\left(\frac{\mathbf{x}-\mathbf{x}_{\mathrm{p}}^{\star}}{\tau}\right) \gamma\left(\beta_{\mathrm{p}}\right) \mathrm{d} \beta_{\mathrm{p}}
$$

where

$$
\mathrm{x}_{\mathrm{p}}^{\star}=\frac{\mathrm{v}^{\star} \tau}{2}\left(\beta_{\mathrm{p}}-\alpha\right)
$$

We can now determine the final distributions:

$$
\begin{aligned}
& \gamma(\beta)=\int \gamma_{\sigma}\left(\beta_{\mathrm{p}}\right) \gamma_{\mathrm{r}}\left(\beta-\beta_{\mathrm{p}}\right) \mathrm{d} \beta_{\mathrm{p}} \\
& \epsilon(\beta)=\int \epsilon_{\sigma}\left(\beta_{\mathrm{p}}\right) \gamma_{\mathrm{r}}\left(\beta-\beta_{\mathrm{p}}\right) \mathrm{d} \beta_{\mathrm{p}}
\end{aligned}
$$

where $\epsilon$ is as per eq. (1).

In the absence of the random process, namely:

$$
\gamma_{\mathrm{r}}\left(\beta-\beta_{\mathrm{p}}\right)=\delta(0)
$$

the final distribution equals the liberation distribution, i.e. the efficiency of the process is $100 \%$.

\section{The Entropy of the Separation Process}

Separation of particles can be considered as a reduction of the entropy of a mixture of the particles. The entropy of a mixture can be expressed as:

$$
\mathrm{S}_{0}=\mathrm{k} \ln \sum \mathrm{C}_{\mathrm{N}}^{\alpha_{\mathrm{N}} \mathrm{N}}
$$

where $N$ is the number of particles and $k$ is the analog of the Boltzmann constant (see eq. (21)). We also have: 


$$
\sum_{\mathbf{i}}^{\mathrm{n}} \alpha_{\mathrm{i}}=1
$$

Applying Stirling formula for sufficiently large $N$, eq. (32) can be rewritten as:

$$
\mathrm{S}_{0}=-\mathrm{kN} \Sigma \alpha_{\mathrm{i}} \ln \alpha_{\mathrm{i}}
$$

and the decrease in entropy following the separation process is given by:

$$
\Delta \mathrm{S}=-\mathrm{kN}\left(\mathrm{S}_{\mathrm{o}}-\sum_{1}^{\mathrm{p}} \gamma_{\mathrm{j}} \sum_{1}^{\mathrm{n}} \beta_{\mathrm{ij}} \ln \beta_{\mathrm{ij}}\right) \leq 0
$$

where $\gamma_{\mathrm{j}}$ is the mass fraction of the $\mathrm{j}$-product, $\beta_{\mathrm{ij}}$ the content of the $\mathrm{i}$-component in the latter. The mass balance yields:

$$
\sum_{j=1}^{p} \gamma_{j} \beta_{i j}=\alpha_{i}
$$

In term of the recovery, eq. (35) can be rewritten as:

$$
\Delta \mathrm{S}=-\mathrm{kN} \sum_{\mathrm{i}=1}^{\mathrm{n}} \alpha_{\mathrm{i}}\left(\ln \alpha_{\mathrm{i}}-\sum_{\mathrm{j}=1}^{\mathrm{p}} \epsilon_{\mathrm{ij}} \ln \beta_{\mathrm{ij}}\right) \leq 0
$$

In a particular case of a two-component mixture $(n=2)$ we have:

$$
\begin{aligned}
& \mathrm{S}_{0}=-\mathrm{kN}[\alpha \ln \alpha+(1-\alpha) \ln (1-\alpha)] \\
& \Delta \mathrm{S}=-\mathrm{kN}\left[\mathrm{S}_{0}-\sum_{\mathrm{j}=1}^{\mathrm{p}} \gamma_{\mathrm{j}}\left(\beta_{\mathrm{j}} \ln \beta_{\mathrm{j}}+\left(1-\beta_{\mathrm{j}}\right) \ln \left(1-\beta_{\mathrm{j}}\right)\right] \leq 0\right.
\end{aligned}
$$

or, in a continuous form:

$$
\Delta \mathrm{S}=-\mathrm{kN}\left[\mathrm{S}_{0}-\int_{0}^{1}(\beta \ln \beta+(1-\beta) \ln (1-\beta)) \mathrm{d} \Gamma\right] \leq 0
$$

From eqs. (35) or (40) we can see that in the case of complete separation (Figure 1) $\Delta S=S_{0}$, i.e. the entropy after separation is $S=0$. In the case of no separation, $\Delta \mathrm{S}=0, \mathrm{~S}=\mathrm{S}_{\mathrm{o}}$ (Figure 2). 


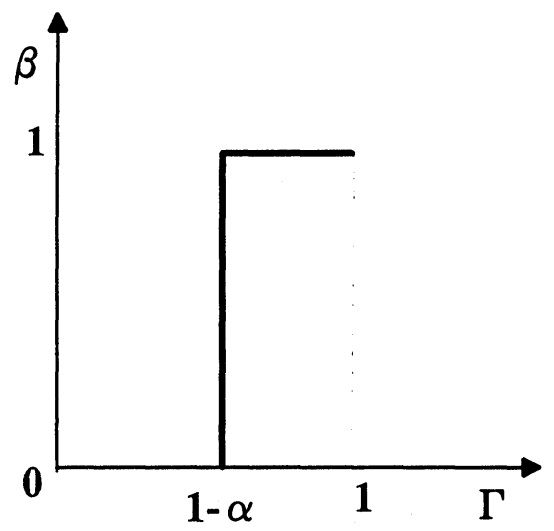

Fig. 1 The case of a complete separation

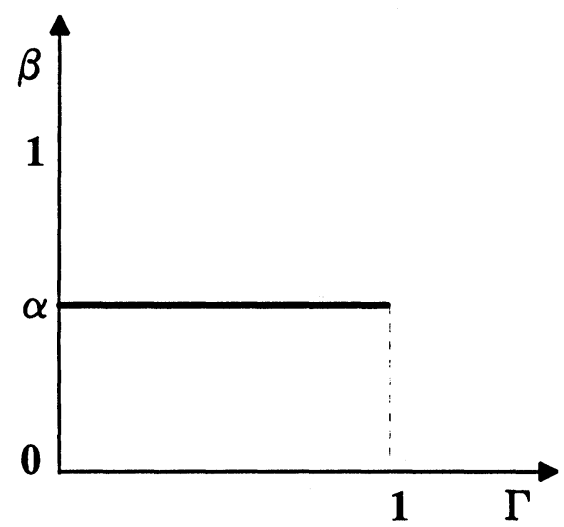

Fig. 2 The case of no separation

The mass balance (the area under the curve) is given by:

$$
\int_{0}^{1} \beta \mathrm{d} \Gamma=\alpha
$$

As noted earlier, the random process is an analog of temperature. According to the third law of thermodynamics (Nernst's theorem) $\left.\mathrm{A}\right|_{\mathrm{T}=0}=0,\left.\mathrm{~S}\right|_{\mathrm{T}=0}=0$, It means that without the random process any small external field would suffice for 
complete separation, for which the separation function $\beta(\Gamma)$ coincides with the liberation function $\beta_{\mathrm{p}}(\Gamma)$.

Let $S_{\mathrm{p}}$ denote the entropy of $\beta(\Gamma)$, then

$$
\Delta S_{\max }=S_{0}-S_{p}
$$

We thus obtain:

$$
\Delta \mathrm{S}(\mathrm{T})=\left\{\begin{array}{l}
\Delta \mathrm{S}_{\mathrm{max}}, \mathrm{T}=0 \\
0, \mathrm{~T}=\infty
\end{array}\right.
$$

and

$$
\Delta S(T)=\left\{\begin{array}{l}
\Delta S_{\text {max }}, T=0 \\
0, A=0
\end{array}\right.
$$

It follows from eqs. (43) and (44) that the entropy difference can be represented as:

$$
\Delta \mathrm{S}=\Delta \mathrm{S}_{\max }\left(1-\frac{1}{\frac{\mathrm{W}_{\mathrm{r}}}{\mathrm{A}}+1}\right)
$$

or, in terms of the efficiency

$$
\eta=\frac{\mathrm{S}-\mathrm{S}_{0}}{\mathrm{~S}_{\max }-\mathrm{S}_{0}}
$$

The initial and final states of the material are related through the following equation:

$$
\begin{aligned}
\eta & =\frac{\left.\left(\int \beta \ln \beta \mathrm{d} \Gamma-\alpha \ln \alpha\right)+(1-\beta) \ln (1-\beta) \mathrm{d} \Gamma-(1-\alpha) \ln (1-\alpha)\right)}{\left(\int \beta_{\mathrm{p}} \ln \beta_{\mathrm{p}} \mathrm{d} \Gamma-\alpha \ln \alpha\right)+\left(\int^{\rho}\left(1-\beta_{\mathrm{p}}\right) \ln \left(1-\beta_{\mathrm{p}}\right) \mathrm{d} \Gamma-(1-\alpha) \ln (1-\alpha)\right)}= \\
& =\frac{1}{1+\frac{\mathrm{v}_{\mathrm{r}}^{2} / \mathrm{v}_{\mathrm{max}}^{\star 2}}{\int\left(\beta_{\mathrm{p}}-\alpha\right)^{2}}}
\end{aligned}
$$


In the case of complete liberation (Fig. 2), eq. (41) is satisfied and we obtain:

$$
\eta=1-\frac{\left(1-\gamma_{\mathrm{c}}\right)\left(\beta_{\mathrm{t}} \ln \beta_{\mathrm{t}}+\left(1-\beta_{\mathrm{c}}\right) \ln \left(1-\beta_{\mathrm{c}}\right)\right)+\gamma_{\mathrm{c}}\left(\beta_{\mathrm{c}} \ln \beta_{\mathrm{c}}+\left(1-\beta_{\mathrm{t}}\right) \ln \left(1-\beta_{\mathrm{t}}\right)\right)}{\alpha \ln \alpha+(1-\alpha) \ln (1-\alpha)}
$$

or, from eq. (47):

$$
\eta=1-\frac{1}{1+\frac{\alpha(1-\alpha)}{\left(\mathrm{v}_{\mathrm{r}}^{\star} / \mathrm{v}_{\max }^{\star}\right)^{2}}}
$$

Particular Cases:

1. No separation: $\beta_{\mathrm{c}}=\beta_{\mathrm{t}}=\alpha, 0<\gamma_{\mathrm{c}}<1, \eta=0$

2. Complete separation: $\beta_{\mathrm{t}}=0, \beta_{\mathrm{c}}=1, \gamma_{\mathrm{c}}=\alpha, \eta=1$

3. The general case: if $\beta_{\mathrm{c}}=\beta_{\mathrm{t}}=\alpha$, then $\eta=0$, and if $\beta(\Gamma)=\beta_{\mathrm{p}}(\Gamma)$ then $\eta=1$.

\section{Example:}

Sylvinite-hallite mixture from the Soligorsk (Belarus) deposit, free-fall electrostatic separation (Fig. 3), complete liberation:

$\alpha=27 \%=0.27$

$\beta_{\mathrm{c}}=62 \%=0.62$ (concentrate)

$\beta_{\mathrm{t}}=3 \%=0.03$ (tailings)

The output of the separator: $M=30 \mathrm{t} / \mathrm{h}=9 \mathrm{~kg} / \mathrm{s}$

Separation angle: $\theta=4^{\circ}, \chi=30^{\circ}$

Height: $\mathrm{H}=1 \mathrm{~m}$

Outlet length: $\ell=1 \mathrm{~m}$

Outlet width: $\delta=0.005 \mathrm{~m}$

From eq. (41): $\gamma_{\mathrm{c}}=0.4, \gamma_{\mathrm{t}}=0.6$

Entropies: $\mathrm{S}_{0} / \mathrm{Nk}=0.62, \mathrm{~S} / \mathrm{Nk}=0.37$

Velocities:

$$
\begin{aligned}
& \mathrm{v}_{\max }=(2 \mathrm{gh})^{\frac{1}{2}}=0.36 \mathrm{~m} / \mathrm{s} \\
& \mathrm{v}_{\mathrm{r}}=\mathrm{M} /(\rho \ell \delta)=0.45 \mathrm{~m} / \mathrm{s}
\end{aligned}
$$

where $\rho$ is the density of the material.

From eq. (49) we then have $\eta=0.38$, while from the entropies we have $\eta=\Delta \mathrm{S} / \mathrm{S}_{0}=0.41$, i.e. practically the same result with the $7.5 \%$ accuracy. 


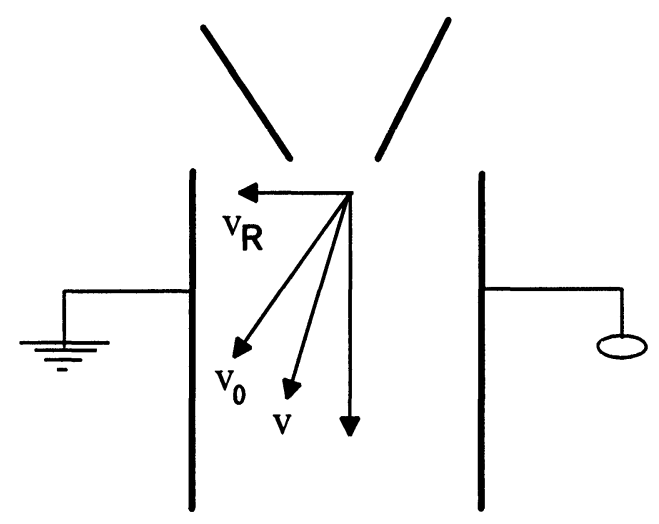

Fig. 3 Schematic diagram of the free-fall electrostatic separator

\section{Practical Application}

Equation (49) shows that the efficiency is a function of the random velocity. With a view to improve the efficiency, we have considered a new design of a feeder with a very small outlet angle (see Fig. 4). From eq. (51) $\mathrm{v}_{\mathrm{r}}$ is also small. The length $a$ should be 0.15 to $0.20 \ell$ since smaller values make for rebound from the feeder walls. Experiments with $\mathrm{NaCl}$ showed an improved efficiency as a result of the modified design.

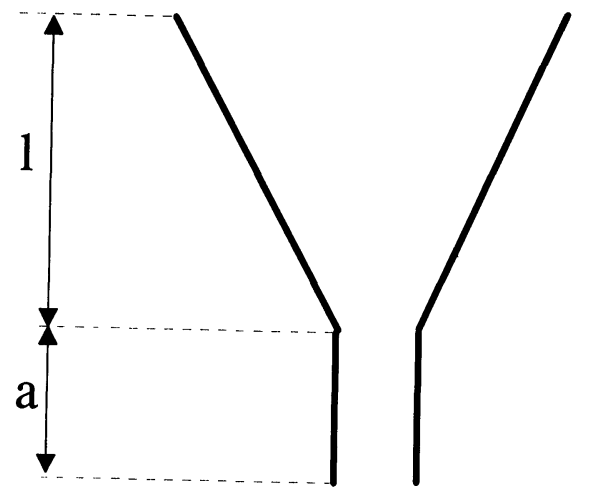

Fig. 4 Schematic diagram of a feeder 


\section{DISCUSSION}

Two approaches to the efficiency of the separation process were considered: in terms of energy and of the entropy difference. The work of the separation force is determined by the spread of the liberation function of the material, and by the properties of the separator.

Losses in the course of the process cause the resultant curve to deviate from the liberation function. This discrepancy can be evaluated as a difference in entropy. On the strength of this thermodynamic analogy, a random process is considered as a source of losses. This is confirmed by the fact of a minimum allowed particle size in any dry separation process.

Thus, a connection has been established between the properties of a material, of a separator and the results of the process. These results can be predicted by comparing the energy of the process and the entropy at the end of the process.

The problem consists of theoretical and practical estimation of the random velocity and of the understanding of its physical meaning. This velocity depends not only on particle size but also on the feeder design. This design should make for a minimised initial velocity in the direction of separation.

\section{CONCLUSION}

The present work offers a physical interpretation of the efficiency of separation and formulae for energy and entropy of the separation processes. This approach permits a prediction of the separation results. Analysis of the electrostatic separation data for sylvinites yields close values for the two criteria.

On the basis of the efficiency/random velocity formula, a new feeder design, suitable for any separation process, was proposed. The solution was experimentally checked with $\mathrm{NaCl}$ and was found to be effective. 


\section{REFERENCES}

[1] A.P. Prosser: Analysis of mineral separation processes. Chem. Proc. Engn., Feb. 1969, pp. 86-88, 96-97

[2] H.B. Barari, P. Sen and P. Gupta: Generalised approach for multicomponent multistage separation efficiency. Trans. SME/AIME 266 (1981), 1901

[3] P.R. Rony: The extent of separation: a universal separation index. Sep. Sci.3 (1968), 239

[4] S. Petho and E. Tompos: Acta Tech. Acad. Sci. Hungar. 78 (1974), 237

[5] K. de Klerk and C.E. Cloete: Entropy as a general separation criterion. Sep. Sci. 6 (1971), 627

[6] E.G. Kelly and D.J. Spottiswood: Introduction to Mineral Processing. John Wiley and Sons (1982)

I.J. Lin: for biography see Magn. Electr. Sep. 3 (1992), 104

A. Katyshev: biography not available

Keywords: efficiency, separation criteria, random process 